\title{
Research Article \\ Schatten Class Toeplitz Operators on the Bergman Space
}

\section{Namita Das}

P. G. Department of Mathematics, Utkal University, Vanivihar, Bhubaneswar, Orissa 751004, India

Correspondence should be addressed to Namita Das, namitadas440@yahoo.co.in

Received 23 July 2009; Revised 7 September 2009; Accepted 14 October 2009

Recommended by Palle Jorgensen

We have shown that if the Toeplitz operator $T_{\phi}$ on the Bergman space $L_{a}^{2}(\mathbb{D})$ belongs to the Schatten class $S_{p}, 1 \leq p<\infty$, then $\tilde{\phi} \in L^{p}(\mathbb{D}, d \lambda)$, where $\tilde{\phi}$ is the Berezin transform of $\phi, d \lambda(z)=$ $d A(z) /\left(1-|z|^{2}\right)^{2}$, and $d A(z)$ is the normalized area measure on the open unit disk $\mathbb{D}$. Further, if $\phi \in L^{p}(\mathbb{D}, d \lambda)$ then $\tilde{\phi} \in L^{p}(\mathbb{D}, d \lambda)$ and $T_{\phi} \in S_{p}$. For certain subclasses of $L^{\infty}(\mathbb{D})$, necessary and sufficient conditions characterizing Schatten class Toeplitz operators are also obtained.

Copyright (C) 2009 Namita Das. This is an open access article distributed under the Creative Commons Attribution License, which permits unrestricted use, distribution, and reproduction in any medium, provided the original work is properly cited.

\section{Introduction}

Let $\mathbb{D}=\{z \in \mathbb{C}:|z|<1\}$ be the open unit disc in the complex plane $\mathbb{C}$. Let $d A(z)=$ $(1 / \pi) d x d y=(1 / \pi) r d r d \theta$ and $L^{2}(\mathbb{D}, d A)$ be the Hilbert space of complex-valued, absolutely square-integrable, Lebesgue measurable functions $f$ on $\mathbb{D}$ with the inner product

$$
\langle f, g\rangle=\int f(z) \overline{g(z)} d A(z)
$$

Let $L^{\infty}(\mathbb{D}, d A)$ denote the Banach space of Lebesgue measurable functions $f$ on $\mathbb{D}$ with

$$
\|f\|_{\infty}=\operatorname{ess} \sup \{|f(z)|: z \in \mathbb{D}\}<\infty
$$

and let $H^{\infty}(\mathbb{D})$ be the space of bounded analytic functions on $\mathbb{D}$. Let $L_{a}^{2}(\mathbb{D})$ be the subspace of $L^{2}(\mathbb{D}, d A)$ consisting of analytic functions. The space $L_{a}^{2}(\mathbb{D})$ is called the Bergman space. Since 
point evaluation at $z \in \mathbb{D}$ is a bounded linear functional on the Hilbert space $L_{a}^{2}(\mathbb{D})$, the Riesz representation theorem implies that there exists a unique function $K_{z}$ in $L_{a}^{2}(\mathbb{D})$ such that

$$
f(z)=\int_{\mathbb{D}} f(w) \overline{K_{z}(w)} d A(w)
$$

for all $f$ in $L_{a}^{2}(\mathbb{D})$. Let $K(z, w)$ be the function on $\mathbb{D} \times \mathbb{D}$ defined by

$$
K(z, w)=\overline{K_{z}(w)}
$$

The function $K(z, w)$ is thus the reproducing kernel for the Bergman space $L_{a}^{2}(\mathbb{D})$ and is called the Bergman kernel. It can be shown that the sequence of functions $\left\{e_{n}(z)\right\}=\left\{\sqrt{n+1} z^{n}\right\}_{n \geq 0}$ forms the standard orthonormal basis for $L_{a}^{2}(\mathbb{D})$ and $K(z, w)=\sum_{n=1}^{\infty} e_{n}(z) \overline{e_{n}(w)}$. The Bergman kernel is independent of the choice of orthonormal basis and $K(z, w)=1 /(1-z \bar{w})^{2}$.

Let $k_{a}(z)=K(z, \bar{a}) / \sqrt{K(\bar{a}, \bar{a})}=\left(1-|a|^{2}\right) /(1-\bar{a} z)^{2}$. These functions $k_{a}$ are called the normalized reproducing kernels of $L_{a}^{2}$; it is clear that they are unit vectors in $L_{a}^{2}$. For any $a \in \mathbb{D}$, let $\phi_{a}$ be the analytic mapping on $\mathbb{D}$ defined by $\phi_{a}(z)=(a-z) /(1-\bar{a} z), z \in \mathbb{D}$. An easy calculation shows that the derivative of $\phi_{a}$ at $z$ is equal to $-k_{a}(z)$. It follows that the real Jacobian determinant of $\phi_{a}$ at $z$ is

$$
J_{\phi_{a}}(z)=\left|k_{a}(z)\right|^{2}=\frac{\left(1-|a|^{2}\right)^{2}}{|1-\bar{a} z|^{4}} .
$$

When $|z| \rightarrow 1, k_{z} \rightarrow 0$ weakly and the normalized reproducing kernels $k_{z}, z \in \mathbb{D}$ span $L_{a}^{2}(\mathbb{D})[1]$. Since $L_{a}^{2}(\mathbb{D})$ is a closed subspace of $L^{2}(\mathbb{D}, d A$ ) (see [1]), there exists an orthogonal projection $P$ from $L^{2}(\mathbb{D}, d A)$ onto $L_{a}^{2}(\mathbb{D})$. For $\phi \in L^{\infty}(\mathbb{D})$, we define the Toeplitz operator $T_{\phi}$ on $L_{a}^{2}(\mathbb{D})$ by $T_{\phi} f=P(\phi f), f \in L_{a}^{2}(\mathbb{D})$. For $\phi \in L^{\infty}(\mathbb{D})$, let $\tilde{\phi}(z)=\left\langle T_{\phi} k_{z}, k_{z}\right\rangle$. The function $\tilde{\phi}(z)$ is called the Berezin transform of $\phi$. Let $H_{\phi}$ be the Hankel operator from $L_{a}^{2}$ into $\left(L_{a}^{2}\right)^{\perp}$ defined by $H_{\phi} f=(I-P)(\phi f)$. It is easy to check that $H_{\phi}^{*} H_{\phi}=T_{|\phi|^{2}}-T_{\bar{\phi}} T_{\phi}$ (see [1]). For $\psi \in L^{\infty}(\mathbb{D})$, define $S_{\psi}: L_{a}^{2} \rightarrow L_{a}^{2}$ as $S_{\psi} f=P J(\psi f)$, where $J: L^{2} \rightarrow L^{2}$ is defined as $J f(z)=f(\bar{z})$. The operator $S_{\psi}$ is called the little Hankel operator on $L_{a}^{2}(\mathbb{D})$. Let $d \lambda(z)=K(z, z) d A(z)=d A(z) /\left(1-|z|^{2}\right)^{2}$, the Mobius invariant measure on $\mathbb{D}$. Let $\mathcal{L}(H)$ be the set of all bounded linear operators from the Hilbert space $H$ into itself, and let $\mathcal{L C}(H)$ be the set of all compact operators in $\mathcal{L}(H)$.

Often it is not easy to verify that a linear operator is bounded, and it is even more difficult to determine its norm. No conditions on the matrix entries $a_{i j}$ have been found which are necessary and sufficient for $A$ to be bounded, nor has $\|A\|$ been determined in the general case. For the more general problem we also need analogues of the notions of operator norm. For more details see $[2,3]$. The family of norms that has received much attention during the last decade is the Schatten norm.

A proper two-sided ideal $\tau$ in $\mathcal{L}(H)$ is said to be a norm ideal if there is a norm on $\tau$ satisfying the following properties:

(i) $\left(\tau,\|\cdot\|_{\tau}\right)$ is a Banach space;

(ii) $\|A X B\|_{\tau} \leq\|A\|\|X\|_{\tau}\|B\|$ for all $A, B \in \mathcal{L}(H)$ and for all $X \in \tau$;

(iii) $\|X\|_{\tau}=\|X\|$ for $X$ a rank one operator. 
If $\left(\tau,\|\cdot\|_{\tau}\right)$ is a norm ideal, then the norm $\|\cdot\|_{\tau}$ is unitarily invariant, in the sense that $\|U A V\|_{\tau}=\|A\|_{\tau}$ for all $A$ in $\tau$ and unitary $U, V$ in $\mathcal{L}(H)$. Each proper ideal of $\mathcal{L}(H)$ is contained in the ideal of compact operators. Two special families of unitarily invariant norms satisfying conditions (i), (ii), and (iii) are the Schatten $p$-norms defined on the set of compact operators and the Ky Fan norms.

For any nonnegative integer $n$, the $n$th singular value of $T \in \mathcal{L C}(H)$ is defined by

$$
s_{n}(T)=\inf \{\|T-K\|: K \in \mathcal{L}(H), \text { rank } K \leq n\} .
$$

Here $\|\cdot\|$ is the operator norm. Clearly, $s_{0}(T)=\|T\|$ and

$$
s_{0}(T) \geq s_{1}(T) \geq s_{2}(T) \geq \cdots \geq 0 .
$$

Thus $s_{n}(T)$ is the distance, with respect to the operator norm, of $T$ from the set of operators of rank at most $n$ in $\mathcal{L}(H)$. The spectral theorem shows that the singular values of the compact operator $T$ are the square roots of the eigenvalues of $T^{*} T$ as long as $H$ is separable and infinite dimensional. Notice that $s_{n}(T)$ can be defined for any $T \in \mathcal{L}(H)$ but clearly, $s_{n}(T) \rightarrow 0$ if and only if $T$ is compact.

The Schatten Von Neumann class $S_{p}=S_{p}(H), 0<p<\infty$, consists of all operators $T \in \mathcal{L C}(H)$ such that

$$
\|T\|_{S_{p}}=\left(\sum_{n=0}^{\infty}\left(S_{n}(T)\right)^{p}\right)^{1 / p}<\infty
$$

If $1 \leq p<\infty$, then $\|\cdot\|_{S_{p}}$ is a norm, which makes $S_{p}$ a Banach space. For $p<1,\|\cdot\|_{S_{p}}$ does not satisfy the triangle inequality, it is a quasinorm (i.e., $\left\|T_{1}+T_{2}\right\|_{S_{p}} \leq C\left(\left\|T_{1}\right\|_{S_{p}}+\left\|T_{2}\right\|_{S_{p}}\right.$ ) for $T_{1}, T_{2} \in S_{p}$ and $C$, a constant), which makes $S_{p}$ a quasiBanach space. We will be mainly concerned with the range $1 \leq p<\infty$. The space $S_{1}$ is also called the trace class and $S_{2}$ is called the Hilbert-Schmidt class. The linear functional trace is defined on $S_{1}$ by

$$
\text { trace } T=\sum_{n=0}^{\infty}\left\langle T \varepsilon_{n}, \varepsilon_{n}\right\rangle, \quad T \in S_{1}
$$

where $\left\{\varepsilon_{n}\right\}_{n \geq 0}$ is an orthonormal basis in $H$. Moreover, the right-hand side does not depend on the choice of the basis. If $1<p<\infty$, the dual space $S_{p}^{*}$ can be identified with $S_{q}$ with respect to the pairing $\langle T, R\rangle=\operatorname{trace} T R^{*}, T \in S_{p}, R \in S_{q}$. Here $q=p /(p-1)$ is the dual exponent. With respect to the same pairing one can identify $S_{1}^{*}$ with $\mathcal{L}(H)$ and $(\mathcal{L C}(H))^{*}$ with $S_{1}$. We refer the reader to $[2,3]$ for basic facts about Schatten $p$-classes. The Schatten $p$-classes should be seen as gradations of compactness for an operator. Each Schatten $p$-class is dense in the space of compact operators in the operator norm. For this reason, it is of interest, given a certain class of operators, to ask whether or not there are compact operators not in any Schatten $p$ class. For instance, this was proved by Arazy et al. [4] and Zhu [1] for Hankel operators on Bergman space.

Hankel operators are closely related to Toeplitz operators. Many problems about Toeplitz operators can also be formulated in terms Hankel operators and vice versa. The 
singular values of Hankel operators on the Hardy space play a crucial role in rational approximation. The celebrated results of Adamjan et al. [5] which give the achievable error in approximating a Hankel operator $\Gamma$ by another one of smaller rank in terms of the singular values of the Hankel operator is an illustration of this. It may be noted here that the Adamjan, Arov, and Krein theorem has had a considerable influence on the treatment of the problem that arises in engineering applications in the context of model reduction, that is, the problem of finding a simple model to replace a relatively complicated one without too great a loss of accuracy. In view of this it would be nice to have a satisfactory characterization for Schatten class Toeplitz operators on the Bergman space.

In this paper we find necessary and sufficient conditions on $\phi$ that will ensure that the Toeplitz operator $T_{\phi}$ belong to $S_{p}, 1 \leq p<+\infty$. This will provide some quantitative estimates (size estimates of these operators) in terms of norms. We will also use $S_{\infty}$ to denote the full algebra of bounded linear operators from the Bergman space $L_{a}^{2}(\mathbb{D})$ into itself.

For $z$ and $w$ in $\mathbb{D}$, let $\phi_{z}(w)=(z-w) /(1-\bar{z} w)$. These are involutive Mobius transformations on $D$. In fact

(1) $\phi_{z} \circ \phi_{z}(w) \equiv w$;

(2) $\phi_{z}(0)=z, \phi_{z}(z)=0$;

(3) $\phi_{z}$ has a unique fixed point in $\mathbb{D}$.

Given $z \in \mathbb{D}$ and $f$ any measurable function on $\mathbb{D}$, we define a function $U_{z} f(w)=$ $k_{z}(w) f\left(\phi_{z}(w)\right)$. Since $\left|k_{z}\right|^{2}$ is the real Jacobian determinant of the mapping $\phi_{z}$ (see [1]), $U_{z}$ is easily seen to be a unitary operator on $L^{2}\left(\mathbb{D}, d A\right.$ ) and $L_{a}^{2}(\mathbb{D})$. It is also easy to check that $U_{z}^{*}=U_{z}$, thus $U_{z}$ is a self-adjoint unitary operator. If $\phi \in L^{\infty}(\mathbb{D}, d A)$ and $z \in \mathbb{D}$ then $U_{z} T_{\phi}=T_{\phi \circ \phi_{z}} U_{z}$. This is because $P U_{z}=U_{z} P$ and for $f \in L_{a}^{2}, T_{\phi \circ \phi_{z}} U_{z} f=T_{\phi \circ \phi_{z}}\left(\left(f \circ \phi_{z}\right) k_{z}\right)=$ $P\left(\left(\phi \circ \phi_{z}\right)\left(f \circ \phi_{z}\right) k_{z}\right)=P\left(U_{z}(\phi f)\right)=U_{z} P(\phi f)=U_{z} T_{\phi} f$. Let Aut $(\mathbb{D})$ be the Lie group of all automorphisms (biholomorphic mappings) of $\mathbb{D}$, and $G_{0}$ the isotropy subgroup at 0 ; that is, $G_{0}=\{\Psi \in \operatorname{Aut}(\mathbb{D}): \Psi(0)=0\}$.

\section{Compact Operators Whose Real and Imaginary Parts Are Positive}

Zhu [1] had shown that if $\phi$ is a nonnegative function on $\mathbb{D}, 1 \leq p \leq \infty$, then $T_{\phi}$ is in the Schatten class $S_{p}$ if and only if $\tilde{\phi}(z)$ is in $L^{p}(\mathbb{D}, d \lambda)$. The following is an easy consequence of it.

Proposition 2.1. Suppose that $T_{\phi} \in \mathcal{L}\left(L_{a}^{2}(\mathbb{D})\right)$ is such that $T_{\phi}=T_{\phi_{1}}+i T_{\phi_{2}}$, where $\phi_{1} \geq 0$ and $\phi_{2} \geq 0$. The Toeplitz operator $T_{\phi} \in S_{p}, 1 \leq p \leq \infty$ if and only if $\tilde{\phi}(z) \in L^{p}(\mathbb{D}, d \lambda)$. In this case, $\left\|T_{\phi}\right\|_{p}^{2} \leq\left\|T_{\phi_{1}}\right\|_{p}^{2}+\left\|T_{\phi_{2}}\right\|_{p}^{2}$ if $2 \leq p<\infty$ and $\left\|T_{\phi}\right\|_{p}^{2} \geq\left\|T_{\phi_{1}}\right\|_{p}^{2}+\left\|T_{\phi_{2}}\right\|_{p}^{2}$ if $1 \leq p \leq 2$.

Proof. Suppose $2 \leq p \leq \infty$ and $T_{\phi} \in S_{p}$. Notice that $T_{\phi_{1}}=\left(T_{\phi}+T_{\phi}^{*}\right) / 2$ and $T_{\phi_{2}}=\left(T_{\phi}-T_{\phi}^{*}\right) / 2 i$ and since $S_{p}$ is a Banach space and is closed under adjoints, hence $T_{\phi_{1}}$ and $T_{\phi_{2}}$ belong to $S_{p}$. From [1], it follows that $\widetilde{\phi_{1}}, \widetilde{\phi_{2}} \in L^{p}(\mathbb{D}, d \lambda)$. Hence $\widetilde{\phi}=\widetilde{\phi_{1}}+i \widetilde{\phi_{2}} \in L^{p}(\mathbb{D}, d \lambda)$. Now suppose $\widetilde{\phi} \in$ $L^{p}(\mathbb{D}, d \lambda), 2 \leq p \leq \infty$. This implies $\widetilde{\phi_{1}}, \widetilde{\phi_{2}} \in L^{p}(\mathbb{D}, d \lambda)$. From [1], it follows that $T_{\phi_{1}}, T_{\phi_{2}} \in S_{p}$. Hence $T_{\phi} \in S_{p}$ as $S_{p}$ is a vector space and $\left\|T_{\phi}\right\|_{p}^{2} \leq\left\|T_{\phi_{1}}\right\|_{p}^{2}+\left\|T_{\phi_{2}}\right\|_{p}^{2}$ (see [6]). Now let $1 \leq p<2$ and assume $T_{\phi} \in S_{p}$. This implies $T_{\phi_{1}}, T_{\phi_{2}} \in S_{p}$ and therefore by [1], $\widetilde{\phi_{1}}, \widetilde{\phi_{2}} \in L^{p}(\mathbb{D}, d \lambda)$. Hence $\tilde{\phi} \in L^{p}(\mathbb{D}, d \lambda)$ and by [6], $\left\|T_{\phi}\right\|_{p}^{2} \geq\left\|T_{\phi_{1}}\right\|_{p}^{2}+\left\|T_{\phi_{2}}\right\|_{p}^{2}$. Now suppose $\tilde{\phi} \in L^{p}(\mathbb{D}, d \lambda), 1 \leq p<2$. Then $\widetilde{\phi_{1}}, \widetilde{\phi_{2}} \in L^{p}(\mathbb{D}, d \lambda)$ and hence $T_{\phi_{1}}, T_{\phi_{2}} \in S_{p}$. Since $S_{p}$ is a vector space, $T_{\phi} \in S_{p}$. 
Example 2.2. Let $\phi(z)=(1-|z|)^{r}, r>0$. Then $T_{\phi} \in S_{2}$ only if $r>1 / 2$, and $T_{\phi} \in S_{1}$ only if $r>1$. This can be seen as follows. The matrix of $T_{\phi}$ with respect to the standard orthonormal basis $\left\{e_{n}(z)\right\}_{n \geq 0}=\left\{\sqrt{n+1} z^{n}\right\}_{n \geq 0}$ of $L_{a}^{2}(\mathbb{D})$ is diagonal, $t_{k k}=\left\langle T_{\phi} e_{k}, e_{k}\right\rangle=(2 k+2) ! /((r+1)(r+$ 2) $\cdots(r+2 k+2))$ and $\sum_{k=0}^{\infty}\left|t_{k k}\right|^{2}=\infty$ for $r \leq 1 / 2$. Similarly $\sum_{k=0}^{\infty} t_{k k}<\infty$ if $r>1$. If $r \leq 1$, $\sum_{k=0}^{\infty} t_{k k}=\infty$.

Proposition 2.3. Let $\phi \in L^{\infty}(\mathbb{D})$ and suppose that $\phi$ is not the zero function. If $T_{\phi}$ is compact then Range $T_{\phi}$ is not closed.

Proof. Since $T_{\phi}$ is compact, hence Range $T_{\phi}$ contains no closed infinite-dimensional subspace of $L_{a}^{2}(\mathbb{D})$. If now Range $T_{\phi}$ is closed then Range $T_{\phi}$ is finite dimensional. That is, $T_{\phi}$ is of finite rank. This implies by [7] that $\phi \equiv 0$. This is a contradiction as $\phi$ is not the zero function.

Recall the following.

Suppose that $A$ is a positive operator on a Hilbert space $H$ and $x$ is unit vector in $H$, then (i) $\left\langle A^{p} x, x\right\rangle \geq\langle A x, x\rangle^{p}$ for all $p \geq 1$; (ii) $\left\langle A^{p} x, x\right\rangle \leq\langle A x, x\rangle^{p}$ for all $0<p \leq 1$. For proof see [1].

Suppose that $\varphi: \mathbb{D} \rightarrow \mathbb{D}$ is analytic. Define the composition operator $C_{\varphi}$ from $L_{a}^{2}(\mathbb{D})$ into itself as $C_{\varphi} f=f \circ \varphi$. It is shown in [1] that $C_{\varphi}$ is a bounded linear operator on $L_{a}^{2}(\mathbb{D})$ and $\left\|C_{\varphi}\right\| \leq(1+|\varphi(0)|) /(1-|\varphi(0)|)$. Given $a \in \mathbb{D}$ and $f$ any measurable function on $\mathbb{D}$, we define the function $C_{a} f$ by $C_{a} f(z)=f\left(\phi_{a}(z)\right)$, where $\phi_{a} \in \operatorname{Aut}(\mathbb{D})$. The map $C_{a}$ is a composition operator on $L_{a}^{2}(\mathbb{D})$.

Proposition 2.4. If $\phi \in L^{\infty}(\mathbb{D})$ then $T_{\phi}$ is compact if and only if $T_{\phi \circ \phi_{z}}$ is compact.

Proof. This follows from the fact that $T_{\phi \circ \phi_{z}}=U_{z} T_{\phi} U_{z}$ and as $U_{z}^{2}=I$.

Proposition 2.5. For $\phi \in L^{\infty}(\mathbb{D}), \operatorname{MO}(\phi)^{2}(z)=\widetilde{|\phi|^{2}}(z)-|\widetilde{\phi}(z)|^{2} \leq\left\|H_{\phi} k_{z}\right\|^{2}+\left\|H_{\bar{\phi}} k_{z}\right\|^{2}$.

Proof. Observe that

$$
\begin{aligned}
\left\|H_{\phi} k_{z}\right\| & =\left\|(I-P)\left(\phi k_{z}\right)\right\| \\
& =\left\|(I-P) U_{z}\left(\phi \circ \phi_{z}\right)\right\| \\
& =\left\|U_{z}(I-P)\left(\phi \circ \phi_{z}\right)\right\| \\
& =\left\|(I-P)\left(\phi \circ \phi_{z}\right)\right\| \\
& =\left\|\phi \circ \phi_{z}-P\left(\phi \circ \phi_{z}\right)\right\| .
\end{aligned}
$$

Similarly, we have $\left\|H_{\bar{\phi}} k_{z}\right\|=\left\|\bar{\phi} \circ \phi_{z}-P\left(\bar{\phi} \circ \phi_{z}\right)\right\|=\left\|\phi \circ \phi_{z}-\overline{P\left(\bar{\phi} \circ \phi_{z}\right)}\right\|$. Since $\tilde{\phi}(z)=P\left(\phi \circ \phi_{z}\right)(0)$ and $P \bar{g}(z)=\bar{g}(0)$ for any $g \in L_{a}^{2}$ and all $z \in \mathbb{D}$, we have

$$
\begin{aligned}
\operatorname{MO}(\phi)^{2}(z) & =\widetilde{|\phi|^{2}}(z)-|\tilde{\phi}(z)|^{2} \\
& =\left\|\phi \circ \phi_{z}-P\left(\phi \circ \phi_{z}\right)(0)\right\|^{2} \\
& =\left\|\phi \circ \phi_{z}-P\left(\phi \circ \phi_{z}\right)\right\|^{2}+\left\|P\left(\phi \circ \phi_{z}\right)-P\left(\phi \circ \phi_{z}\right)(0)\right\|^{2}
\end{aligned}
$$




$$
\begin{aligned}
& =\left\|H_{\phi} k_{z}\right\|^{2}+\left\|P\left(\phi \circ \phi_{z}\right)-\overline{P\left(\bar{\phi} \circ \phi_{z}\right)(0)}\right\|^{2} \\
& =\left\|H_{\phi} k_{z}\right\|^{2}+\left\|P\left(\phi \circ \phi_{z}-\overline{P\left(\bar{\phi} \circ \phi_{z}\right)}\right)\right\|^{2} \\
& \leq\left\|H_{\phi} k_{z}\right\|^{2}+\left\|\phi \circ \phi_{z}-\overline{P\left(\bar{\phi} \circ \phi_{z}\right)}\right\|^{2} \\
& =\left\|H_{\phi} k_{z}\right\|^{2}+\left\|H_{\bar{\phi}} k_{z}\right\|^{2} .
\end{aligned}
$$

Let $h>1$. The generalized Kantorvich constant $K(p)$ is defined by

$$
K(p)=\frac{h^{p}-h}{(p-1)(h-1)}\left(\frac{p-1}{p} \frac{h^{p}-1}{h^{p}-h}\right)^{p}
$$

for any real number $p$ and it is known that $K(p) \in(0,1]$ for $p \in[0,1]$. We state below the known results on the generalized Kantorvich constant $K(p)$. Let $A$ be strictly positive operator satisfying $M I \geq A \geq m I>0$, where $M>m>0$. Put $h=M / m>1$. Then the following [8] inequalities (2.4) and (2.5) hold for every unit vector $x$ and are equivalent:

$$
\begin{gathered}
K(p)\langle A x, x\rangle^{p} \geq\left\langle A^{p} x, x\right\rangle \geq\langle A x, x\rangle^{p} \quad \text { for any } p>1 \text { or any } p<0 ; \\
\langle A x, x\rangle^{p} \geq\left\langle A^{p} x, x\right\rangle \geq K(p)\langle A x, x\rangle^{p} \quad \text { for any } p \in(0,1] .
\end{gathered}
$$

The Kantorvich constant $K(p)$ is symmetric with respect to $p=1 / 2$ and $K(p)$ is an increasing function of $p$ for $p \geq 1 / 2, K(p)$ is a decreasing function of $p$ for $p \leq 1 / 2$, and $K(0)=K(1)=1$. Further, $K(p) \geq 1$ for $p \geq 1$ or $p \leq 0$, and $1 \geq K(p) \geq 2 h^{1 / 4} /\left(h^{1 / 2}+1\right)$ for $p \in[0,1]$.

Proposition 2.6. Let $T_{\phi}$ be strictly positive satisfying $M I \geq T_{\phi} \geq m I>0$, where $M>m>0$. The following hold.

(i) If $0<p<\infty$ and $T_{\phi} \in S_{p}$ then $\tilde{\phi} \in L^{p}(\mathbb{D}, d \lambda)$.

(ii) If $0<p \leq 1, \tilde{\phi} \in L^{p}(\mathbb{D}, d \lambda)$ then $T_{\phi} \in S_{p}$.

(iii) Let $p \in[1, \infty)$ be such that $K(p)<\infty$. If $\tilde{\phi} \in L^{p}(\mathbb{D}, d \lambda)$ then $T_{\phi} \in S_{p}$.

Proof. Suppose $p>1$ and $T_{\phi} \in S_{p}$. Then

$$
\int_{\mathbb{D}}\left\langle T_{\phi}^{p} k_{z}, k_{z}\right\rangle d \lambda(z)=\int_{\mathbb{D}}\left\langle\left|T_{\phi}\right|^{p} k_{z}, k_{z}\right\rangle d \lambda(z)<\infty
$$

Hence by (2.4), $\int_{\mathbb{D}}\left\langle T_{\phi} k_{z}, k_{z}\right\rangle^{p} d \lambda(z)<\infty$. That is, $\tilde{\phi} \in L^{p}(\mathbb{D}, d \lambda)$. Suppose $0<p \leq 1$ and $T_{\phi} \in S_{p}$. Then $\int_{\mathbb{D}}\left\langle T_{\phi}^{p} k_{z}, k_{z}\right\rangle d \lambda(z)=\int_{\mathbb{D}}\left\langle\left|T_{\phi}\right|^{p} k_{z}, k_{z}\right\rangle d \lambda(z)<\infty$. Hence from (2.5), it follows that $K(p) \int_{\mathbb{D}}\left\langle T_{\phi} k_{z}, k_{z}\right\rangle^{p} d \lambda(z)<\infty$. Since $K(p) \in(0,1]$ for $p \in[0,1]$, hence $\tilde{\phi} \in L^{p}(\mathbb{D}, d \lambda)$. 
Now assume $\tilde{\phi} \in L^{p}(\mathbb{D}, d \lambda)$. Then if $0<p \leq 1$ then by (2.5), we have $\int_{\mathbb{D}}\left\langle\left|T_{\phi}\right|^{p} k_{z}, k_{z}\right\rangle d \lambda(z)<\infty$ and hence $T_{\phi} \in S_{p}$. If $1 \leq p<\infty$, then by (2.4) and (2.5), if $K(p)<\infty$ and $\tilde{\phi} \in L^{p}(\mathbb{D}, d \lambda)$ then $\int_{\mathbb{D}}\left\langle\left|T_{\phi}\right|^{p} k_{z}, k_{z}\right\rangle d \lambda(z)<\infty$ and $T_{\phi} \in S_{p}$.

\section{Schatten Class Operators}

In this section, we will obtain conditions to describe Schatten class Toeplitz operators on Bergman space $L_{a}^{2}(\mathbb{D})$. The results of this paper hold for Bergman spaces $L_{a}^{2}(\Omega)$, where $\Omega$ is any bounded symmetric domain in $\mathbb{C}$. For simplicity, we consider only the case of the open unit disk $\mathbb{D}$ in $\mathbb{C}$.

Let $B T=\left\{f \in L^{1}:\|f\|_{B T}=\sup _{z \in \mathbb{D}}|\widetilde{f}|(z)<\infty\right\}$. The space $L^{\infty}$ is properly contained in $B T$ (see [9]) and if $\phi \in B T$ then $T_{\phi}$ is bounded on $L_{a}^{2}$ and there is a constant $C$ such that $\left\|T_{\phi}\right\| \leq C\|\phi\|_{B T}$.

Theorem 3.1. Suppose $1 \leq p<\infty$ and $d \lambda(z)=d A(z) /\left(1-|z|^{2}\right)^{2}$. Then the following hold. (1) If $T_{\phi} \in S_{p}$, then $\tilde{\phi} \in L^{p}(\mathbb{D}, d \lambda)$. (2) If $\phi \in L^{p}(\mathbb{D}, d \lambda)$ then $\tilde{\phi} \in L^{p}(\mathbb{D}, d \lambda)$ and $T_{\phi} \in S_{p}$.

Proof. Suppose $T_{\phi} \in S_{p}$. Then

$$
\int_{\mathbb{D}}\left\langle\left|T_{\phi}\right|^{p} k_{w}, k_{w}\right\rangle d \lambda(w)<\infty
$$

That is, $\int_{\mathbb{D}}\left\langle\left(T_{\phi}^{*} T_{\phi}\right)^{p / 2} k_{w}, k_{w}\right\rangle d \lambda(w)<\infty$. If $2 \leq p<\infty$, then

$$
\int_{\mathbb{D}}\left\langle T_{\phi}^{*} T_{\phi} k_{w}, k_{w}\right\rangle^{p / 2} d \lambda(w) \leq \int_{\mathbb{D}}\left\langle\left(T_{\phi}^{*} T_{\phi}\right)^{p / 2} k_{w}, k_{w}\right\rangle d \lambda(w)<\infty
$$

This implies

$$
\begin{aligned}
\int_{\mathbb{D}}\left\|P\left(\phi \circ \phi_{w}\right)\right\|^{p} d \lambda(w) & =\int_{\mathbb{D}}\left\|P\left(U_{w}\left(\phi k_{w}\right)\right)\right\|^{p} d \lambda(w) \\
& =\int_{\mathbb{D}}\left\|U_{w} T_{\phi} k_{w}\right\|^{p} d \lambda(w) \\
& =\int_{\mathbb{D}}\left\|T_{\phi} k_{w}\right\|^{p} d \lambda(w) \\
& =\int_{\mathbb{D}}\left\langle T_{\phi}^{*} T_{\phi} k_{w}, k_{w}\right\rangle^{p / 2} d \lambda(w)<\infty .
\end{aligned}
$$

Now $\left|P\left(\phi \circ \phi_{w}\right)(0)\right|=\left|\left\langle P\left(\phi \circ \phi_{w}\right), 1\right\rangle\right|=\left|\left\langle U_{w}\left(T_{\phi} k_{w}\right), 1\right\rangle\right|=\left|\left\langle T_{\phi} k_{w}, U_{w} 1\right\rangle\right|=\left|\left\langle T_{\phi} k_{w}, k_{w}\right\rangle\right| \leq$ $\left\|T_{\phi} k_{w}\right\|=\left\|P\left(\phi \circ \phi_{w}\right)\right\|$. Thus $\int_{\mathbb{D}}\left|P\left(\phi \circ \phi_{w}\right)(0)\right|^{p} d \lambda(w)<\infty$. That is, $\int_{\mathbb{D}}|\tilde{\phi}(w)|^{p} d \lambda(w)<$ $\infty$ and $\tilde{\phi} \in L^{p}(\mathbb{D}, d \lambda)$. Suppose $1 \leq p<2$. Then by Heinz inequality [10], 
it follows that

$$
\begin{aligned}
\infty & >\int_{\mathbb{D}}\left\langle\left|T_{\phi}\right|^{p} k_{w}, k_{w}\right\rangle d \lambda(w) \\
& =\int_{\mathbb{D}}\left\langle\left|T_{\phi}\right|^{2 \cdot p / 2} k_{w}, k_{w}\right\rangle d \lambda(w) \\
& \geq \int_{\mathbb{D}} \frac{\left|\left\langle T_{\phi} k_{w}, k_{w}\right\rangle\right|^{2}}{\left\langle\left|T_{\phi}^{*}\right|^{2(1-p / 2)} k_{w}, k_{w}\right\rangle} d \lambda(w) \\
& =\int_{\mathbb{D}} \frac{|\tilde{\phi}(w)|^{2}}{\left\|P\left(\bar{\phi} \circ \phi_{w}\right)\right\|^{2-p}} d \lambda(w) \\
& =\int_{\mathbb{D}}|\tilde{\phi}(w)|^{2}\left\|P\left(\bar{\phi} \circ \phi_{w}\right)\right\|^{p-2} d \lambda(w) \\
& \geq \int_{\mathbb{D}} \frac{|\tilde{\phi}(w)|^{2}}{\left\|P\left(\bar{\phi} \circ \phi_{w}\right)\right\|^{2}\left\|P\left(\bar{\phi} \circ \phi_{w}\right)\right\|^{p} d \lambda(w)} \\
& \geq \int_{\mathbb{D}} \frac{|\tilde{\phi}(w)|^{2}}{C^{2}\|\phi\|_{B T}^{2}}\left|P\left(\phi \circ \phi_{w}\right)(0)\right|^{p} d \lambda(w) \\
& =\int_{\mathbb{D}} \frac{|\tilde{\phi}(w)|^{2}}{C^{2}\|\phi\|_{B T}^{2}}|\tilde{\phi}(w)|^{p} d \lambda(w),
\end{aligned}
$$

since

$$
\begin{aligned}
\left\langle\left|T_{\phi}^{*}\right|^{2-p} k_{w}, k_{w}\right\rangle & =\left\langle\left|T_{\phi}^{*}\right|^{2 \cdot(2-p) / 2} k_{w}, k_{w}\right\rangle \\
& \leq\left\langle\left|T_{\phi}^{*}\right|^{2} k_{w}, k_{w}\right\rangle^{(2-p) / 2} \\
& =\left\langle T_{\phi} T_{\phi}^{*} k_{w}, k_{w}\right\rangle^{(2-p) / 2} \\
& =\left\|T_{\phi}^{*} k_{w}\right\|^{2-p} \\
& =\left\|P\left(\bar{\phi} \circ \phi_{w}\right)\right\|^{2-p} .
\end{aligned}
$$

Hence

$$
\int_{\mathbb{D}}|\tilde{\phi}(w)|^{p+2} d \lambda(w)<\infty
$$

and therefore $\int_{\mathbb{D}}|\tilde{\phi}(w)|^{p} d \lambda(w)<\infty$. Thus $\tilde{\phi} \in L^{p}(\mathbb{D}, d \lambda)$. 
Now suppose $\phi \in L^{1}(\mathbb{D}, d \lambda)$. Then the change of the order of integration

$$
\begin{aligned}
\int_{\mathbb{D}}|\tilde{\phi}(w)| d \lambda(w) & =\int_{\mathbb{D}}|\tilde{\phi}(w)| \frac{d A(w)}{\left(1-|w|^{2}\right)^{2}} \\
& \leq \int_{\mathbb{D}}\left(\int_{\mathbb{D}}|\phi(z)| \frac{\left(1-|w|^{2}\right)^{2}}{|1-\overline{\mathbf{w}} z|^{4}} d A(z)\right) \frac{d A(w)}{\left(1-|w|^{2}\right)^{2}} \\
& =\int_{\mathbb{D}}|\phi(z)| \int_{\mathbb{D}|1-\overline{\mathbf{w}} z|^{4}} \frac{d A(w)}{\left|z(z)=\int_{\mathbb{D}}\right| \phi(z) \mid\left\langle k_{z}, k_{z}\right\rangle d A(z)} \\
& =\int_{\mathbb{D}}|\phi(z)| \frac{d A(z)}{\left(1-|z|^{2}\right)^{2}}
\end{aligned}
$$

is justified by the positivity of the integrand. Hence $\tilde{\phi} \in L^{1}(\mathbb{D}, d \lambda)$. Similarly if $\phi \in L^{\infty}(\mathbb{D})$ then $\tilde{\phi} \in L^{\infty}(\mathbb{D})$ as $|\tilde{\phi}(w)|=\left|\left\langle\phi k_{w}, k_{w}\right\rangle\right| \leq\left\|\phi k_{w}\right\|_{2}\left\|k_{w}\right\|_{2} \leq\|\phi\|_{\infty}\left\|k_{w}\right\|_{2}^{2}=\|\phi\|_{\infty}$. By Marcinkiewicz interpolation theorem it follows that if $\phi \in L^{p}(\mathbb{D}, d \lambda)$ then $\tilde{\phi} \in L^{p}(\mathbb{D}, d \lambda)$ for $1 \leq p \leq \infty$. Now suppose $\phi \in L^{p}(\mathbb{D}, d \lambda), 1 \leq p \leq \infty$. We will prove $T_{\phi} \in S_{p}$. The case $p=+\infty$ is trivial. By interpolation we need only to prove the result for $p=1$. Suppose $\phi \in L^{1}(\mathbb{D}, d \lambda)$ and $\left\{e_{n}\right\}=\left\{\sqrt{n+1} z^{n}\right\}_{n=0}^{\infty}$ is the standard orthonormal basis for $L_{a}^{2}(\mathbb{D})$. Now $\left\langle T_{\phi} e_{n}, e_{n}\right\rangle=\int_{\mathbb{D}}\left|e_{n}(z)\right|^{2} \phi(z) d A(z)$ and

$$
\begin{aligned}
\sum_{n=0}^{\infty}\left|\left\langle T_{\phi} e_{n}, e_{n}\right\rangle\right| & \leq \int_{\mathbb{D}_{n}=0} \sum_{n}^{\infty}\left|e_{n}(z)\right|^{2}|\phi(z)| d A(z) \\
& \leq \int_{\mathbb{D}} K(z, z)|\phi(z)| d A(z) \\
& =\int_{\mathbb{D}}|\phi(z)| d \lambda(z) .
\end{aligned}
$$

Thus $T_{\phi} \in S_{1}$ and $\left\|T_{\phi}\right\|_{S_{1}} \leq \int_{\mathbb{D}}|\phi(z)| d \lambda(z)$.

It is not so difficult to verify the conditions in Theorem 3.1.

Example 3.2. Let $\Phi(z)=\left(1-|z|^{2}\right) \log \left(1-|z|^{2}\right)$. Then $\Phi \in L^{2}(\mathbb{D}, d \lambda)$. This can be verified as follows:

$$
\begin{aligned}
\int_{\mathbb{D}}\left(1-|z|^{2}\right)^{2}\left|\log \left(1-|z|^{2}\right)\right|^{2} d \lambda(z) \\
\quad=\int_{\mathbb{D}}\left(1-|z|^{2}\right)^{2}\left|\log \left(1-|z|^{2}\right)\right|^{2} \frac{d A(z)}{\left|\left(1-|z|^{2}\right)\right|^{2}}
\end{aligned}
$$




$$
\begin{aligned}
& =\int_{\mathbb{D}}\left|\log \left(1-|z|^{2}\right)\right|^{2} d A(z) \\
& =\int_{0}^{1}\left|\log \left(1-r^{2}\right)\right|^{2} 2 r d r \\
& =\int_{0}^{1}|\log (1-t)|^{2} d t \\
& =\int_{0}^{1}|\log t|^{2} d t
\end{aligned}
$$

and changing the variable to $y=-\log t$, this reduces to $\int_{0}^{\infty} y^{2} e^{-y} d y=\Gamma(3)=2<\infty$. Thus $T_{\Phi} \in S_{2}$.

Example 3.3. Let $g(z)=\ln |z|^{2}$. Then

$$
\begin{aligned}
\int_{\mathbb{D}}|g(z)|^{2} d \lambda(z) & =\int_{0}^{1}\left(\frac{\ln t}{1-t}\right)^{2} d t \\
& =\int_{0}^{1} \sum_{n=0}^{\infty} \sum_{m=0}^{\infty} t^{m+n} \ln ^{2} t d t \\
& =\sum_{n=0}^{\infty} \sum_{m=0}^{\infty} \frac{2}{(m+n+1)^{3}} \\
& =\sum_{k=0}^{\infty} \frac{2}{(k+1)^{2}} \\
& =\frac{\pi^{2}}{3}<\infty .
\end{aligned}
$$

Thus $g \in L^{2}(\mathbb{D}, d \lambda)$. Direct computation reveals that $\tilde{g}(x)=|x|^{2}-1$ and $\tilde{g} \in L^{2}(\mathbb{D}, d \lambda)$.

Example 3.4. Let $\phi(z)=1-|z|$. Then by Example 2.2, $T_{\phi} \in S_{2}$ and

$$
\begin{aligned}
\int_{\mathbb{D}}|\phi(z)|^{2} d \lambda(z) & =\int_{\mathbb{D}}(1-|z|)^{2} \frac{d A(z)}{\left(1-|z|^{2}\right)^{2}} \\
& =\int_{\mathbb{D}} \frac{d A(z)}{(1+|z|)^{2}} \\
& \leq \int_{\mathbb{D}} d A(z)=1 .
\end{aligned}
$$

Thus $\phi \in L^{2}(\mathbb{D}, d \lambda)$ and hence $\tilde{\phi} \in L^{2}(\mathbb{D}, d \lambda)$. 
It may be noted that the space $L^{p}(\mathbb{D}, d \lambda), 1 \leq p<\infty$, contains no nonzero harmonic functions and even no nonzero constants. To see this, for example, for $L^{2}(\mathbb{D}, d \lambda)$, let

$$
M(r)=\frac{1}{2 \pi} \int_{0}^{2 \pi}\left|f\left(r e^{i t}\right)\right|^{2} d t
$$

This is a nonnegative and nondecreasing function of $r$ and $\|f\|_{L^{2}(\mathbb{D}, d \lambda)}^{2}=\int_{0}^{1} M(r)(2 r /(1-$ $\left.\left.r^{2}\right)^{2}\right) d r<\infty$. So $M(r)$ must tend to 0 as $r \rightarrow 1$. Thus $M(r) \equiv 0$, and therefore $f \equiv 0$.

But although there is no nonzero harmonic functions in $L^{2}(\mathbb{D}, d \lambda)$, there are plenty of subharmonic functions. Consider the function $f(z)=\ln |z|^{2}$. We have verified in Example 3.3 that $f \in L^{2}(\mathbb{D}, d \lambda)$. Suppose that $f$ is real-valued subharmonic and $f \in L^{2}(\mathbb{D}, d \lambda)$. The subharmonicity of $f$ implies that $\widetilde{f}(w)=\int_{\mathbb{D}} f\left(\phi_{w}(z)\right) d A(w) \geq f\left(\phi_{w}(0)\right)=f(w)$. Hence $\tilde{f} \geq f$. Let $\Delta_{h}:=\left(1-|z|^{2}\right)^{2}\left(\partial^{2} / \partial z \partial \overline{\mathbf{z}}\right)$. It can be verified that $\Delta_{h}\left(f \circ \phi_{a}\right)=\left(\Delta_{h} f\right) \circ \phi_{a}$ and note that $\Delta_{h} \tilde{f}=\widetilde{\Delta_{h} f} \geq 0$ since $\Delta_{h} f \geq 0$. In other words, $\tilde{f}$ is also subharmonic. Proceeding by induction, if we define $B g=\widetilde{g}$ on $L^{2}(\mathbb{D}, d \lambda)$ then we obtain $B^{n} f$ is subharmonic for all $n \in \mathbb{N}$ and $\left\{B^{n} f\right\}_{n \in \mathbb{N}}$ is a nondecreasing sequence of functions.

Corollary 3.5. If $\phi \in h^{\infty}(\mathbb{D})$, the space of bounded harmonic functions on $\mathbb{D}$ and $1 \leq p<\infty$, then $T_{\phi} \in S_{p}$ if and only if $\phi \equiv 0$.

Proof. The proof of the corollary follows from the above discussion and the fact [1] that $\phi \in$ $h^{\infty}(\mathbb{D})$ if and only if $\tilde{\phi}=\phi$.

Corollary 3.6. If $\phi$ is a real-valued bounded subharmonic function on $\mathbb{D}, 1 \leq p<\infty$, then $T_{\phi} \in S_{p}$ if and only if $\tilde{\phi} \in L^{p}(\mathbb{D}, d \lambda)$.

Proof. By Theorem 3.1, if $T_{\phi} \in S_{p}$ then $\tilde{\phi} \in L^{p}(\mathbb{D}, d \lambda)$. Conversely if $\phi$ is real-valued, subharmonic, bounded on $\mathbb{D}$ and $\tilde{\phi} \in L^{p}(\mathbb{D}, d \lambda)$ then $\tilde{\phi}$ is also subharmonic and the subharmonicity of $\phi$ implies that $\tilde{\phi}(w)=\int_{\mathbb{D}} \phi\left(\phi_{w}(z)\right) d A(z) \geq \phi\left(\phi_{w}(0)\right)=\phi(w)$. Hence $\int_{\mathbb{D}}|\tilde{\phi}(w)|^{p} d \lambda(w)=\int_{\mathbb{D}}|\phi(w)|^{p} d \lambda(w)$ as $\phi \in L^{p}(\mathbb{D}, d \lambda)$ implies $\tilde{\phi} \in L^{p}(\mathbb{D}, d \lambda)$ and the result follows from Theorem 3.1.

Corollary 3.7 follows immediately from Corollary 3.5. We present a proof of Corollary 3.7 to show a different method of approach.

Corollary 3.7. If $\phi \in H^{\infty}(\mathbb{D}) \cup \overline{H^{\infty}(\mathbb{D})}$ then the Toeplitz operator $T_{\phi} \in S_{p}, 1 \leq p<\infty$ if and only if $\phi \equiv 0$.

Proof. Notice that if $\phi \in H^{\infty}(\mathbb{D}) \cup \overline{H^{\infty}(\mathbb{D})}$ then the following two possibilities hold.

$\left(^{*}\right)$ For all $z \in \mathbb{D}$, either $P\left(\phi \circ \phi_{z}\right) \neq P\left(\phi \circ \phi_{z}\right)(0)$ or $P\left(\bar{\phi} \circ \phi_{\mathbf{z}}\right) \neq P\left(\bar{\phi} \circ \phi_{\mathbf{z}}\right)(0)$.

$\left.{ }^{(* *}\right)$ There exists $z \in D$ such that $P\left(\phi \circ \phi_{z}\right)=P\left(\phi \circ \phi_{z}\right)(0)$ and $P\left(\bar{\phi} \circ \phi_{\mathbf{z}}\right)=P\left(\bar{\phi} \circ \phi_{\mathbf{z}}\right)(0)$.

Suppose that $\left({ }^{* *}\right)$ holds and let $z \in \mathbb{D}$ be such that $P\left(\phi \circ \phi_{z}\right)=P\left(\phi \circ \phi_{z}\right)(0)$ and $P\left(\bar{\phi} \circ \phi_{\mathbf{z}}\right)=P\left(\bar{\phi} \circ \phi_{\mathbf{z}}\right)(0)$. Then this implies $T_{\phi} k_{z}=c_{z} k_{z}$ and $T_{\bar{\phi}} k_{z}=\overline{\mathbf{c}}_{\mathbf{z}} k_{z}$. Hence $T_{\phi}$ has an eigenvalue. From [1], it follows that $\phi$ is a constant and $T_{\phi}$ is not compact unless $\phi \equiv 0$. 
Now suppose that $\left(^{*}\right)$ holds, $1 \leq p<\infty$. Then for all $z \in \mathbb{D},\left\|H_{\phi} k_{z}\right\|^{2}+\left\|H_{\bar{\phi}} k_{z}\right\|^{2}=$ $\left\|\phi \circ \phi_{z}-P\left(\phi \circ \phi_{z}\right)\right\|^{2}+\left\|\bar{\phi} \circ \phi_{z}-P\left(\bar{\phi} \circ \phi_{z}\right)\right\|^{2}<\left\|\phi \circ \phi_{z}-P\left(\phi \circ \phi_{z}\right)(0)\right\|^{2}+\left\|\bar{\phi} \circ \phi_{z}-P\left(\bar{\phi} \circ \phi_{z}\right)(0)\right\|^{2}=$ $2\left(\widetilde{|\phi|^{2}}(z)-|\tilde{\phi}(z)|^{2}\right)$. Let $c_{z}=\left(\left\|H_{\phi} k_{z}\right\|^{2}+\left\|H_{\bar{\phi}} k_{z}\right\|^{2}\right) /\left(\left.\left|\widetilde{\left.\phi\right|^{2}}(z)-\right| \tilde{\phi}(z)\right|^{2}\right)$. Note that $1 \leq c_{z}<2$ for all $z \in \mathbb{D}$. It follows from Proposition 2.5 and from the previous discussion.

Notice $T_{\phi}$ compact implies that $T_{\phi}^{*} T_{\phi}=T_{|\phi|^{2}}-H_{\phi}^{*} H_{\phi}$ is compact. Thus $0 \leq\left\langle T_{\phi}^{*} T_{\phi} k_{z}, k_{z}\right\rangle=$ $\widetilde{|\phi|^{2}}(z)-\left\|H_{\phi} k_{z}\right\|^{2} \rightarrow 0$ as $|z| \rightarrow 1^{-}$. Similarly since $T_{\phi} T_{\phi}^{*}$ is compact, $0 \leq \widetilde{|\phi|^{2}}(z)-\left\|H_{\bar{\phi}} k_{z}\right\|^{2} \rightarrow 0$ as $|z| \rightarrow 1^{-}$.

Thus $0 \leq 2 \widetilde{|\phi|^{2}}(z)-\left(\left\|H_{\phi} k_{z}\right\|^{2}+\left\|H_{\bar{\phi}} k_{z}\right\|^{2}\right) \rightarrow 0$ as $|z| \rightarrow 1^{-}$. Hence it follows that $0 \leq 2 \widetilde{|\phi|^{2}}(z)-c_{z}\left(\widetilde{|\phi|^{2}}(z)-|\tilde{\phi}(z)|^{2}\right)=2 \widetilde{|\phi|^{2}}(z)-\left(\left\|H_{\phi} k_{z}\right\|^{2}+\left\|H_{\bar{\phi}} k_{z}\right\|^{2}\right) \rightarrow 0$ as $|z| \rightarrow 1^{-}$. So $0 \leq\left.\left(2-c_{z}\right)\left|\widetilde{\left.\phi\right|^{2}}(z)+c_{z}\right| \tilde{\phi}(z)\right|^{2} \rightarrow 0$ as $|z| \rightarrow 1^{-}$, where $1 \leq c_{z}<2$. Since $T_{\phi}$ is compact, $|\tilde{\phi}(z)| \rightarrow 0$ as $|z| \rightarrow 1^{-}$. Thus $\widetilde{|\phi|^{2}}(z) \rightarrow 0$ as $|z| \rightarrow 1^{-}$. Similarly since $T_{\phi \circ \phi_{z}}$ is compact we can show that $\left|\widetilde{\phi \circ \phi_{z}}\right|^{2}(z) \rightarrow 0$ as $|z| \rightarrow 1^{-}$. Thus

$$
\int_{\mathbb{D}}\left|\left(\phi \circ \phi_{z}\right)(w)\right|^{2}\left|k_{z}(w)\right|^{2} d A(w) \longrightarrow 0
$$

as $|z| \rightarrow 1^{-}$. Hence $\int_{\mathbb{D}}|\phi(w)|^{2} d A(w)=0$ as $U_{z} k_{z}=k_{z}\left(\phi_{z}(w)\right) k_{z}(w)=1$. It follows therefore that $\phi(w)=0$ almost everywhere and hence $\phi \equiv 0$. Therefore, $T_{\phi} \equiv 0$.

Let $\tilde{T}(z)=\left\langle T k_{z}, k_{z}\right\rangle$ for $T \in \mathcal{L}\left(L_{a}^{2}(\mathbb{D})\right)$. It follows from Heinz inequality [10] that

$$
\left|\left\langle T_{\phi} k_{z}, k_{z}\right\rangle\right|^{2} \leq\left\langle\left|T_{\phi}\right|^{2 \alpha} k_{z}, k_{z}\right\rangle\left\langle\left|T_{\phi}^{*}\right|^{2(1-\alpha)} k_{z}, k_{z}\right\rangle
$$

for all $z \in \mathbb{D}$ and $0 \leq \alpha \leq 1$. That is, $|\tilde{\phi}(z)|^{2} \leq \widetilde{\left|T_{\phi}\right|^{2 \alpha}}(z) \mid \widetilde{\left.T_{\phi}^{*}\right|^{2(1-\alpha)}}(z)$ for all $z \in \mathbb{D}$. Hence if $\widetilde{\left|T_{\phi}\right|^{2 \alpha}} \in L^{p}(\mathbb{D}, d \lambda)$ and $\widetilde{\left.T_{\phi}^{*}\right|^{2(1-\alpha)}} \in L^{q}(\mathbb{D}, d \lambda), 1 / p+1 / q=1$, it follows from Holders inequality that $\tilde{\phi} \in L^{2}(\mathbb{D}, d \lambda)$. From Theorem 3.1, it follows that $T_{\tilde{\phi}} \in S_{2}$.

We say $T$ majorizes $S \in \mathcal{L}\left(L_{a}^{2}(\mathbb{D})\right)$ if $\|S f\| \leq M\|T f\|$ for all $f \in L_{a}^{2}(\mathbb{D})$.

Corollary 3.8. If $\phi \geq 0, \tilde{\phi} \in L^{p}(\mathbb{D}, d \lambda), 1 \leq p \leq 2$, and $T_{\phi}$ majorizes $T_{\psi}$ then $T_{\psi} \in S_{p}$.

Proof. Since $T_{\phi}$ majorizes $T_{\psi}$, it follows that $\left\|T_{\psi} f\right\| \leq M\left\|T_{\phi} f\right\|$ for some $M>0$ and for all $f \in L_{a}^{2}$. Hence $\left\langle T_{\psi}^{*} T_{\psi} f, f\right\rangle \leq M^{2}\left\langle T_{\phi}^{*} T_{\phi} f, f\right\rangle$ for all $f \in L_{a}^{2}$. That is, $T_{\psi}^{*} T_{\psi} \leq M^{2} T_{\phi}^{*} T_{\phi}$. Since $1 \leq p \leq 2$, we obtain from $[10,11]$ that $\left(T_{\psi}^{*} T_{\psi}\right)^{p / 2} \leq M^{p}\left(T_{\phi}^{*} T_{\phi}\right)^{p / 2}$. That is, $\left|T_{\psi}\right|^{p} \leq M^{p}\left|T_{\phi}\right|^{p}$ for $1 \leq p \leq 2$. Now if $\tilde{\phi} \in L^{p}(\mathbb{D}, d \lambda), \phi \geq 0$, then by [1], $T_{\phi} \in S_{p}$ and $\int_{\mathbb{D}}\left\langle\left|T_{\phi}\right|^{p} k_{z}, k_{z}\right\rangle d \lambda(z)<\infty$. Hence $\int_{\mathbb{D}}\left\langle\left|T_{\psi}\right|^{p} k_{z}, k_{z}\right\rangle d \lambda(z)<\infty$ and $T_{\psi} \in S_{p}$.

Corollary 3.9. Let $\phi, \psi \in L^{\infty}(\mathbb{D})$. If Range $T_{\bar{\psi}} \subseteq$ Range $T_{\bar{\phi}}$ and $\phi \in L^{p}(\mathbb{D}, d \lambda), 1 \leq p \leq 2$ then $T_{\psi} \in S_{p}$ and $\tilde{\psi} \in L^{p}(\mathbb{D}, d \lambda)$.

Proof. Range $T_{\bar{\psi}} \subseteq$ Range $T_{\bar{\phi}}$ implies that $T_{\phi}$ majorizes $T_{\psi}$. Hence $\left\|T_{\psi} f\right\| \leq M\left\|T_{\phi} f\right\|$ for some $M>0$ and for all $f \in L_{a}^{2}$. Hence $\left\langle T_{\psi}^{*} T_{\psi} f, f\right\rangle \leq M^{2}\left\langle T_{\phi}^{*} T_{\phi} f, f\right\rangle$ for all $f \in L_{a}^{2}$. That is, $T_{\psi}^{*} T_{\psi} \leq$ $M^{2} T_{\phi}^{*} T_{\phi}$. Since $1 \leq p \leq 2$, we obtain from $[10,11]$ that $\left(T_{\psi}^{*} T_{\psi}\right)^{p / 2} \leq M^{p}\left(T_{\phi}^{*} T_{\phi}\right)^{p / 2}$. That is, 
$\left|T_{\psi}\right|^{p} \leq M^{p}\left|T_{\phi}\right|^{p}$ for $1 \leq p \leq 2$. Now if $\phi \in L^{p}(\mathbb{D}, d \lambda)$ then $T_{\phi} \in S_{p}$ and $\int_{\mathbb{D}}\left\langle\left|T_{\phi}\right|^{p} k_{z}, k_{z}\right\rangle d \lambda(z)<\infty$. Hence $\int_{\mathbb{D}}\left\langle\left|T_{\psi}\right|^{p} k_{z}, k_{z}\right\rangle d \lambda(z)<\infty$. Thus $T_{\psi} \in S_{p}$ and $\tilde{\psi} \in L^{p}(\mathbb{D}, d \lambda)$.

Definition 3.10. A function $G \in L_{a}^{2}(D)\left(G \in H^{2}\right)$ is called an inner function [12] in $L_{a}^{2}(D)$ (resp., $H^{2}$ ) if $|G|^{2}-1$ is orthogonal to $H^{\infty}$. If for some inner function $G \in L_{a}^{2}(D)$ the following conditions hold then we call it a finite zero divisor in $L_{a}^{2}(D)$. (i) $G$ vanishes on $\mathbf{a}=\left\{a_{j}\right\}_{j=1}^{N^{N}}$, a finite sequence of points in $D$. (ii) $\|G\|_{L^{2}}=1$. (iii) $G$ is equal to a constant plus a linear combination of the Bergman kernel functions $K\left(z, a_{1}\right), K\left(z, a_{2}\right), \ldots, K\left(z, a_{n}\right)$ and certain of their derivatives. (iv) $|G|^{2}-1$ is orthogonal to $L_{h^{\prime}}^{1}$ the class of harmonic functions in $L^{1}$ of the disc.

Corollary 3.11. Suppose $\phi \in L^{\infty}(\mathbb{D})$ and $\operatorname{ker} T_{\phi} \subseteq G L_{a}^{2}$, where $G$ is a finite inner divisor in $L_{a}^{2}(\mathbb{D})$. If $\phi \in L^{p}(\mathbb{D}, d \lambda), 1 \leq p \leq 2$, then $S_{\psi} \in S_{p}$, where $S_{\psi}$ is the little Hankel operator on $L_{a}^{2}(\mathbb{D})$ such that $\operatorname{ker} S_{\psi}=G L_{a}^{2}$.

Proof. Let $\mathbf{b}=\left(b_{j}\right)_{j=1}^{N}$ be a finite set of points in $\mathbb{D}$ that are the zeroes of the finite inner divisor $G$ counting multiplicities and $\supset=I(\mathbf{b})=\left\{f \in L_{a}^{2}(D): f=0\right.$ on $\left.\mathbf{b}\right\}$. Then $G$ is the solution of the extremal problem

$$
\sup \left\{\operatorname{Re} f^{(n)}(0): f \in \mathcal{O},\|f\|_{L^{2}} \leq 1\right\},
$$

where $n$ is the number of times zero appears in the sequence $\mathbf{b}$ (i.e., the functions in $\partial$ have a common zero of order $n$ at the origin). It is shown in [12] that for

$$
\bar{\psi}=\sum_{j=1}^{N} \sum_{v=0}^{m_{j}-1} c_{j v} \frac{\partial^{v}}{\partial \bar{b}_{j}^{v}} K_{b_{j}}(z),
$$

where $c_{j v} \neq 0$ for all $j, v$ and $m_{j}$ is the number of times $b_{j}$ appear in $\mathbf{b}, \operatorname{ker} S_{\psi}=G L_{a}^{2}(D)$. Now $\operatorname{ker} T_{\phi} \subseteq G L_{a}^{2}=\operatorname{ker} S_{\psi}$ implies [13] the operator $T_{\phi}$ that majorizes $S_{\psi}$. Since $\phi \in L^{p}(\mathbb{D}, d \lambda)$, $1 \leq p \leq 2, T_{\phi} \in S_{p}$. By similar arguments as in Corollary 3.9 one can show that $S_{\psi} \in S_{p}$.

For any $a \in \mathbb{D}$, let $\gamma_{a}$ be the unique geodesic such that $\gamma_{a}(0)=0, \gamma_{a}(1)=a$. Then there exists a unique $\phi_{a} \in \operatorname{Aut}(\mathbb{D})$ such that $\phi_{a} \circ \phi_{a}(z) \equiv z$ and $\gamma_{a}(1 / 2)$ is an isolated fixed point of $\phi_{a}$. Further $\phi_{a}$ is the geodesic symmetry at $\gamma_{a}(1 / 2)$. We denote by $m_{a}$ the geodesic midpoint $\gamma_{a}(1 / 2)$ of 0 and $a$. It can easily be verified that each $\phi_{a}$ has $m_{a}$ as a unique fixed point and for $a \in \mathbb{D}, m_{a}=\left(\left(1-\sqrt{1-|a|^{2}}\right) /|a|^{2}\right) a$.

Given $\lambda \in \mathbb{D}$ and a measurable function $f$ on $\mathbb{D}$, we have $f \circ \phi_{\lambda}=f$ if and only if there exists an even function $g$ on $\mathbb{D}$ such that $f=g \circ \phi_{m_{\lambda}} ; f \circ \phi_{\lambda}=-f$ if and only if there exists an odd function $g$ on $\mathbb{D}$ such that $f=g \circ \phi_{m_{\lambda}}$. For proof of this fact see [14].

Corollary 3.12. If $\phi=\theta \circ \phi_{m_{z}}$ for some $z \in \mathbb{D}, \theta \in L^{\infty}(\mathbb{D})$ and where $\theta$ is an even function and $T_{\phi} \in S_{p}, 1 \leq p<\infty$, then

$$
\left\|T_{\psi \circ \phi_{z}-\psi+\phi}\right\|_{S_{p}} \geq\left\|T_{\phi}\right\|_{S_{p}}
$$

for all $T_{\psi} \in S_{p}$. 
Proof. Since $\phi=\theta \circ \phi_{m_{z}}$ where $\theta$ is an even function, by [14], we have $\phi \circ \phi_{z}=\phi$. Hence $U_{z} T_{\phi} U_{z}=T_{\phi \circ \phi_{z}}=T_{\phi}$. As $U_{z}$ is unitary, from [15], it follows that

$$
\left\|U_{z} T_{\psi} U_{z}-T_{\psi}+T_{\phi}\right\|_{S_{p}} \geq\left\|T_{\phi}\right\|_{S_{p}}
$$

for all $T_{\psi} \in S_{p}$. Hence

$$
\left\|T_{\psi \circ \phi_{z}-\psi+\phi}\right\|_{S_{p}} \geq\left\|T_{\phi}\right\|_{S_{p}}
$$

for all $T_{\psi} \in S_{p}$.

Theorem 3.13. Suppose there exist constants $C, M>0$ such that $C>M$ and $\left\|P\left(\phi \circ \phi_{z}\right)-C\right\| \leq M$ for all $z \in \mathbb{D}, \tilde{\phi} \in L^{p}(\mathbb{D}, d \lambda)$ and $p \in[1, \infty)$ is such that the Kantorvich constant $K(p) \in(0, \infty)$. If $T_{\phi}$ is bounded below then $T_{\phi} \in S_{p}$.

Proof. If there exist $C>M>0$ such that for all $z \in \mathbb{D}$, we have $\left\|P\left(\phi \circ \phi_{z}\right)-C\right\| \leq M$ then $\left\|T_{\phi} k_{z}-C k_{z}\right\|=\left\|U_{z} P\left(\phi \circ \phi_{z}\right)-C U_{z} 1\right\|=\left\|P\left(\phi \circ \phi_{z}\right)-C\right\| \leq M$ for all $z \in \mathbb{D}$. Let $\Gamma=C+M$ and $\gamma=C-M$. Then $0<\gamma<\Gamma$ and $C=(\Gamma+\gamma) / 2$ and $M=(\Gamma-\gamma) / 2$. Thus for all $z \in \mathbb{D},\left\|T_{\phi} k_{z}-((\Gamma+\gamma) / 2) k_{z}\right\| \leq(1 / 2)|\Gamma-\gamma|\left\|k_{z}\right\|$. By [16], it follows that there exists $L>0$ such that $|\tilde{\phi}(z)|=\left|\left\langle T_{\phi} k_{z}, k_{z}\right\rangle\right| \geq L\left\|T_{\phi} k_{z}\right\|$ for all $z \in \mathbb{D}$. Notice that the reproducing kernels $\left\{k_{z}: z \in \mathbb{D}\right\}$ span $L_{a}^{2}(\mathbb{D})$ and $T_{\phi}$ is bounded below. Hence there exist $r>0, s>0$ such that $s \leq T_{\phi}^{*} T_{\phi} \leq r$. Now if $\widetilde{\phi} \in L^{p}(\mathbb{D}, d \lambda)$ and $p \in[1, \infty)$ is such that the Kantorvich constant $K(p) \in(0, \infty)$ then $\int_{\mathbb{D}}\left\|T_{\phi} k_{z}\right\|^{p} d \lambda(z)<\infty$. From inequalities (2.4) and (2.5), it follows that $\int_{\mathbb{D}}\left\langle\left|T_{\phi}\right|^{p} k_{z}, k_{z}\right\rangle d \lambda(z)<\infty$. Hence $T_{\phi} \in S_{p}$.

Let $L^{2}(\mathbb{T})$ be the usual Lebesgue space of functions on the unit circle $\mathbb{T}$, and let the Hardy space $H^{2}$ be the class of all $L^{2}(\mathbb{T})$ functions whose negative Fourier coefficients are zero. Let $L^{\infty}(\mathbb{T})$ be the algebra of essentially bounded complex valued functions on the unit circle $\mathbb{T}$ and $H^{\infty}(\mathbb{T})$ be the subalgebra of $L^{\infty}(\mathbb{T})$ consisting of functions whose negative Fourier coefficients are zero. Let $R_{n}$ denote the rational functions with at most $n$ poles (counting multiplicities) all of which are in the interior of $\mathbb{T}$, and let $C(\mathbb{T})$ denote the algebra of continuous functions on $\mathbb{T}$. Notice that the following inclusion relations hold:

$$
H^{\infty}(\mathbb{T}) \subset H^{\infty}(\mathbb{T})+\mathcal{R}_{1} \subset H^{\infty}(\mathbb{T})+\mathcal{R}_{2} \subset \cdots H^{\infty}(\mathbb{T})+C(\mathbb{T})
$$

For $\phi \in L^{\infty}(\mathbb{T})$, let $B_{\phi}: H^{2} \rightarrow H^{2}$ be the Toeplitz operator on $H^{2}$ with symbol $\phi$, and let $\Gamma_{\phi}: H^{2} \rightarrow H^{2}$ be the Hankel operator with symbol $\phi$. Nehari [17], Adamjan et al. [5], and Hartman [18], respectively, proved the following results:

(i) $\left\|\Gamma_{\phi}\right\|=d\left(\phi, H^{\infty}(\mathbb{T})\right)$;

(ii) $s_{k}\left(\Gamma_{\phi}\right)=d\left(\phi, H^{\infty}(\mathbb{T})+\mathcal{R}_{n}\right)$;

(iii) $\left\|\Gamma_{\phi}\right\|_{e}=d\left(\phi, H^{\infty}(\mathbb{T})+C(\mathbb{T})\right)$, 
where $\left\|\Gamma_{\phi}\right\|_{e}$ denotes the essential norm of $\Gamma_{\phi}$. Feintuch in $[19,20]$ obtained the following operator theoretic analogues of these distance formulae. Given $T \in \mathcal{L}\left(H^{2}(\mathbb{T})\right)$, define a sequence $\left\{\Gamma_{n}(\mathbb{T})\right\}$ of operators on $H^{2}(\mathbb{T})$ by $\Gamma_{n}(\mathbb{T})=J_{n} T S^{n+1}, n=1,2, \ldots$, where for $z \in \mathbb{T}$,

$$
J_{n} z^{i}= \begin{cases}z^{n-i}, & \text { if } 0 \leq i \leq n, \\ 0, & \text { if } i>n,\end{cases}
$$

and $S$ is the unilateral shift on $H^{2}(\mathbb{T})$. Let $\hat{\tau}$ denote the family of operators $T$ for which $\left\{\Gamma_{n}(\mathbb{T})\right\}$ converges strongly and

$$
\begin{aligned}
& \mathcal{L}_{0}=\left\{T \in \hat{\mathcal{C}}: \Gamma_{n}(T) \text { converges strongly to } 0\right\} ; \\
& \mathcal{L}_{k}=\mathcal{L}_{0}+\left\{B_{\phi}: \phi \in H^{\infty}(\mathbb{T})+\mathcal{R}_{k}\right\} ; \\
& \mathcal{L}_{\infty}=\mathcal{L}_{0}+\left\{B_{\phi}: \phi \in H^{\infty}(\mathbb{T})+C(\mathbb{T})\right\} .
\end{aligned}
$$

Then (i) $d\left(T, \mathcal{L}_{0}\right)=\|\Gamma(\mathbb{T})\|$; (ii) $d\left(T, \mathcal{L}_{k}\right)=s_{k}(\Gamma(\mathbb{T}))$; (iii) $d\left(T, \mathcal{L}_{\infty}\right)=\|\Gamma(\mathbb{T})\|_{e}$. In particular when $T=B_{\phi}, \phi \in L^{\infty}(\mathbb{T})$ we have

(i) $d\left(B_{\phi}, \mathcal{L}_{0}\right)=\left\|\Gamma_{\phi}\right\|=d\left(\phi, H^{\infty}(\mathbb{T})\right)$;

(ii) $d\left(B_{\phi}, \mathcal{L}_{k}\right)=s_{k}\left(\Gamma_{\phi}\right)=d\left(\phi, H^{\infty}(\mathbb{T})+\mathcal{R}_{k}\right)$;

(iii) $d\left(B_{\phi}, \mathcal{L}_{\infty}\right)=\left\|\Gamma_{\phi}\right\|_{e}=d\left(\phi, H^{\infty}(\mathbb{T})+C(\mathbb{T})\right)$.

Yamada [21] also obtained distance formulas involving the norm of Toeplitz operators on the Hardy space.

It is well known that [1] there are no compact Toeplitz operators on the Hardy space other than the zero operator. In the Bergman space setting, however, there are lots of nontrivial compact Toeplitz operators belonging to different Schatten classes. In view of this it is of interest to know whether such distance formulae is possible for Toeplitz and Hankel operators defined on the Bergman space and the characterization of Schatten class Toeplitz operators is also important in this context. These results play an important role in approximation theory.

\section{References}

[1] K. H. Zhu, Operator Theory in Function Spaces, vol. 139 of Monographs and Textbooks in Pure and Applied Mathematics, Marcel Dekker, New York, NY, USA, 1990.

[2] I. C. Gohberg and M. G. Kreln, Introduction to the Theory of Linear Nonselfadjoint Operators in Hilbert Space, American Mathematical Society, Providence, RI, USA, 1969.

[3] M. S. Birman and M. Z. Solomyak, Spectral Theory of Self-Adjoint Operators in Hilbert Space, Reidel, Dordrecht, The Netherlands, 1986.

[4] J. Arazy, S. D. Fisher, and J. Peetre, "Hankel operators on weighted Bergman spaces," American Journal of Mathematics, vol. 110, no. 6, pp. 989-1053, 1988.

[5] V. M. Adamjan, D. Z. Arov, and M. G. KreĬn, "Analytic properties of Schmidt pairs for a Hankel operator and the generalized Schur-Takagi problem," Mathematics of the USSR-Sbornik, vol. 15, no. 1, pp. 31-73, 1971.

[6] R. Bhatia and X. Zhan, "Compact operators whose real and imaginary parts are positive," Proceedings of the American Mathematical Society, vol. 129, no. 8, pp. 2277-2281, 2001. 
[7] D. H. Luecking, "Finite rank Toeplitz operators on the Bergman space," Proceedings of the American Mathematical Society, vol. 136, no. 5, pp. 1717-1723, 2008.

[8] T. Furuta, "Reverse inequalities involving two relative operator entropies and two relative entropies," Linear Algebra and Its Applications, vol. 403, pp. 24-30, 2005.

[9] J. Miao and D. Zheng, "Compact operators on Bergman spaces," Integral Equations and Operator Theory, vol. 48, no. 1, pp. 61-79, 2004.

[10] E. Heinz, "On an inequality for linear operators in a Hilbert space," in Report of an International Conference on Operator Theory and Group Representations, National Academy of Sciences-National Research Council no. 387, pp. 27-29, Arden House, Harriman, NY, USA, 1955.

[11] T. Furuta, "A simplified proof of Heinz inequality and scrutiny of its equality," Proceedings of the American Mathematical Society, vol. 97, no. 4, pp. 751-753, 1986.

[12] N. Das, "The kernel of a Hankel operator on the Bergman space," The Bulletin of the London Mathematical Society, vol. 31, no. 1, pp. 75-80, 1999.

[13] B. A. Barnes, "Majorization, range inclusion, and factorization for bounded linear operators," Proceedings of the American Mathematical Society, vol. 133, no. 1, pp. 155-162, 2005.

[14] K. H. Zhu, "On certain unitary operators and composition operators," in Operator Theory: Operator Algebras and Applications, vol. 51 of Proceedings of Symposia in Pure Mathematics, Part 2, pp. 371-385, American Mathematical Society, Providence, RI, USA, 1990.

[15] A. Mazouz, "On the range and the kernel of the operator $X \mapsto A X B-X$, , Proceedings of the American Mathematical Society, vol. 127, no. 7, pp. 2105-2107, 1999.

[16] S. S. Dragomir, "Reverses of Schwarz, triangle and Bessel inequalities in inner product spaces," Journal of Inequalities in Pure and Applied Mathematics, vol. 5, no. 3, article 76, pp. 1-18, 2004.

[17] Z. Nehari, “On bounded bilinear forms," Annals of Mathematics, vol. 65, pp. 153-162, 1957.

[18] P. Hartman, "On completely continuous Hankel matrices," Proceedings of the American Mathematical Society, vol. 9, pp. 862-866, 1958.

[19] A. Feintuch, "On Hankel operators associated with a class of non-Toeplitz operators," Journal of Functional Analysis, vol. 94, no. 1, pp. 1-13, 1990.

[20] A. Feintuch, "On asymptotic Toeplitz and Hankel operators," in The Gohberg Anniversary Collection, Vol. II, vol. 41 of Operator Theory: Advances and Applications, pp. 241-254, Birkhäuser, Basel, Switzerland, 1989.

[21] M. Yamada, Distance Formulas of Asymptotic Toeplitz and Hankel Operators, Hokkaido University Preprint Series in Mathematics no. 182, 1993. 


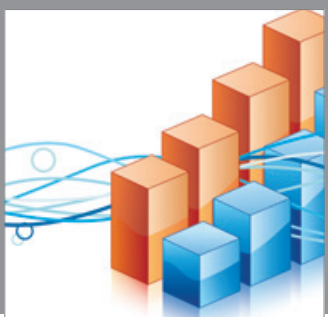

Advances in

Operations Research

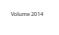

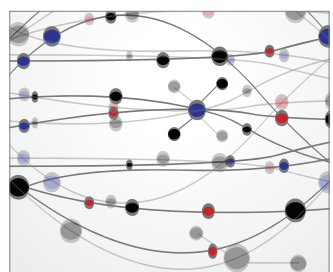

\section{The Scientific} World Journal
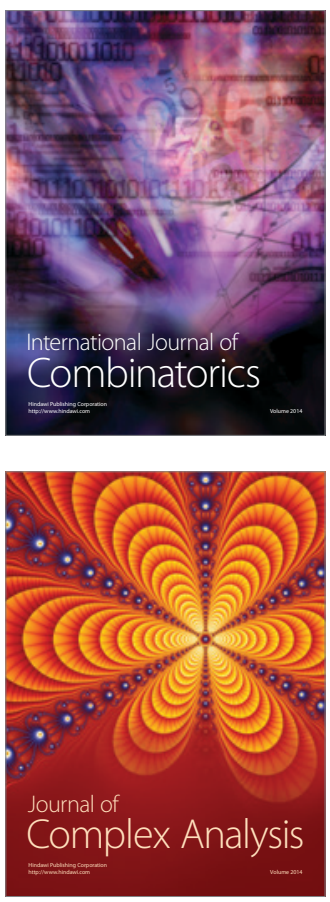

International Journal of

Mathematics and

Mathematical

Sciences
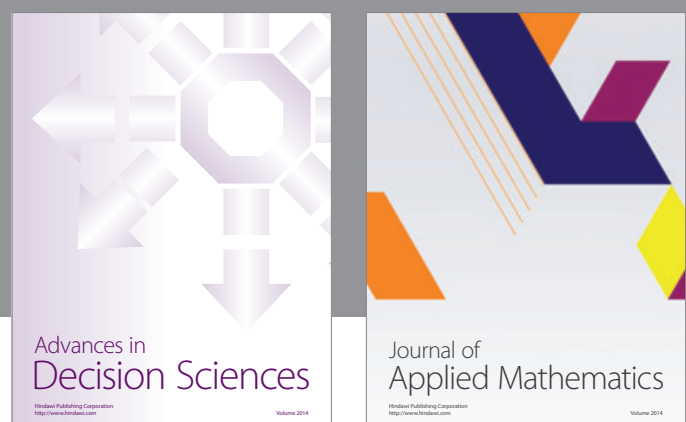

Journal of

Applied Mathematics
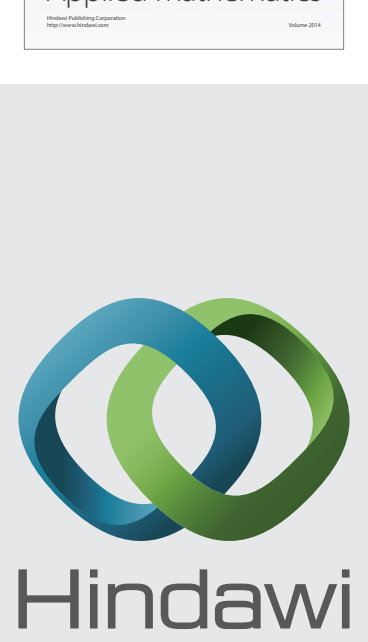

Submit your manuscripts at http://www.hindawi.com
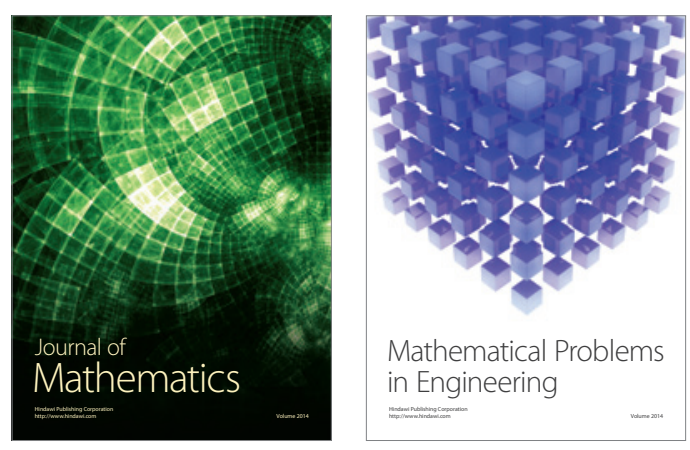

Mathematical Problems in Engineering
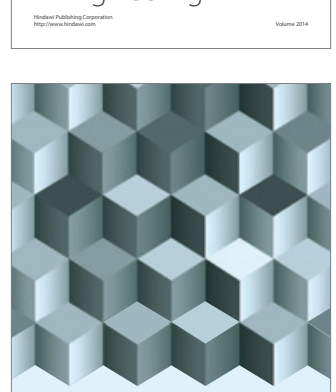

Journal of

Function Spaces
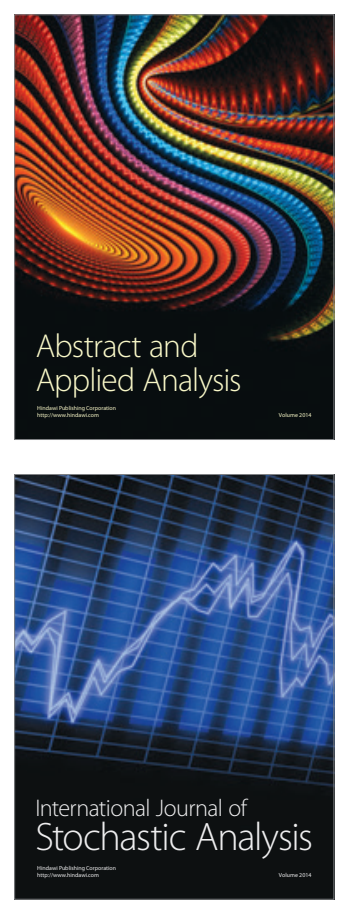

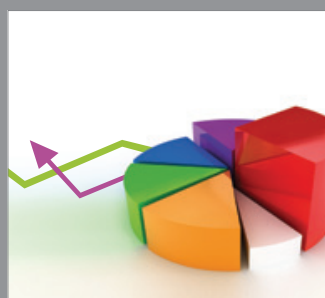

ournal of

Probability and Statistics

Promensencen
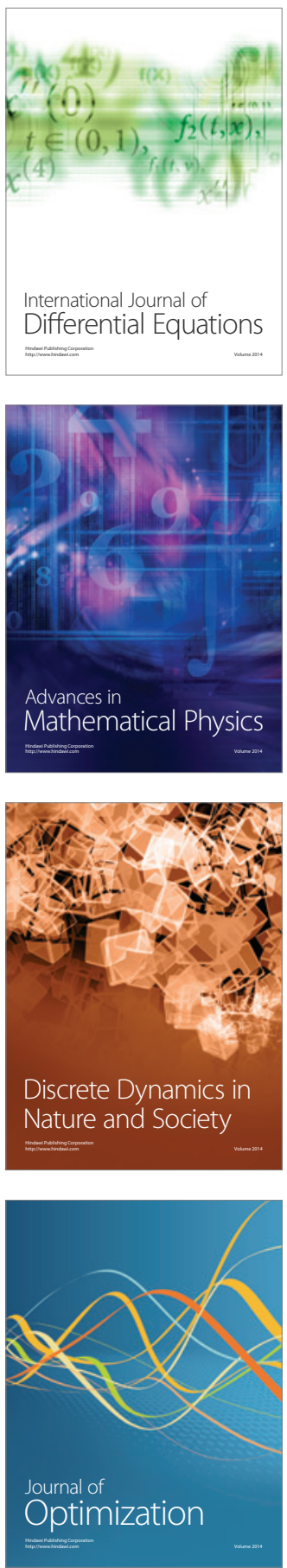